\title{
Systematic epistatic mapping of cellular processes
}

Maximilian Billmann ${ }^{1,3}$ and Michael Boutros ${ }^{1,2^{*}}$ (D)

\begin{abstract}
Genetic screens have identified many novel components of various biological processes, such as components required for cell cycle and cell division. While forward genetic screens typically generate unstructured 'hit'lists, genetic interaction mapping approaches can identify functional relations in a systematic fashion. Here, we discuss a recent study by our group demonstrating a two-step approach to first screen for regulators of the mitotic cell cycle, and subsequently guide hypothesis generation by using genetic interaction analysis. The screen used a high-content microscopy assay and automated image analysis to capture defects during mitotic progression and cytokinesis. Genetic interaction networks derived from process-specific features generate a snapshot of functional gene relations in those processes, which follow a temporal order during the cell cycle. This complements a recently published approach, which inferred directional genetic interactions reconstructing hierarchical relationships between genes across different phases during mitotic progression. In conclusion, this strategy leverages unbiased, genome-wide, yet highly sensitive and process-focused functional screening in cells.
\end{abstract}

Keywords: Genetic interactions, Image analysis, RNAi, Cell cycle

\section{Background}

During cell division, a cell undergoes several consecutive events to replicate and divide its genome and distribute it to two daughter cells. The temporal order and mechanism of those events has been extensively explored using methods that visualize the DNA content or size of cells [1] or their content of cell cycle-specific proteins such as cyclins [2]. Microscopy techniques illustrate the localization of cellular components [3-5] and cell cycle specific factors or the presence of cell cycle markers such as residue-specific phosphorylation of histone H3 [6, 7]. Image analysis algorithms can capture such cellular structures in an automated fashion. For example, this enables quantification of the fraction of cells with condensed chromosomes or visible serine 10 phosphorylated histone $\mathrm{H} 3$ (pH3) levels as a proxy for cell cycle defects. Importantly, image analysis can extract multiple phenotypic features from a cell population, which allows for simultaneously following distinct biological processes. For instance,

\footnotetext{
*Correspondence: m.boutros@dkfz.de

2 German Cancer Consortium (DKTK), 69120 Heidelberg, Germany

Full list of author information is available at the end of the article
}

automated analysis of cells stained for their DNA and $\mathrm{pH} 3$ visualizes perturbations causing defects in mitotic progression (increased mitotic index in the cell population) or cytokinesis (increased average nuclear size) (Fig. 1).

To identify regulators of the cell cycle in a systematic fashion, model systems such as budding yeast, cultured Drosophila or human cells have been exploited in genome-scale functional screens [8-10]. Advanced automated image analysis have enabled screening for modulators of diverse biological processes [11-13]. For instance, an RNAi screen with live imaging of human HeLa cells has exploited a stably expressed GFP-labeled histone H2B to identify genes required for proper chromosome segregation and cell cycle propagation [13]. While such studies have identified cell cycle regulators with high sensitivity, genetic interaction analysis approaches have been able to define functional and epistatic relations between genes [14]. Genetic interaction analysis systematically exploits genetic buffering by genetic variants, which completely or partially overlap in function [14-17]. Genetic interaction studies have been performed in yeast and assayed cell fitness as a composite phenotype $[14,16]$ capturing 


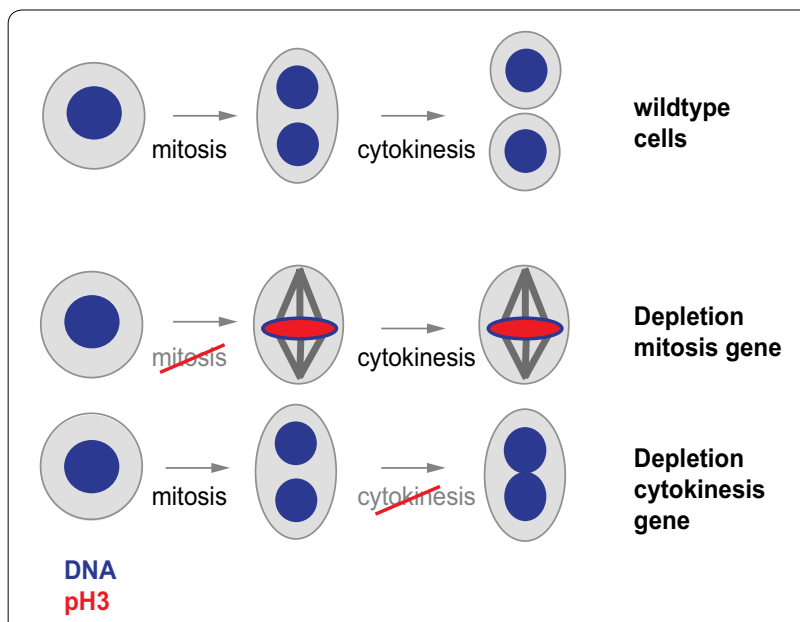

Fig. 1 Schematic illustration of a cell progressing through mitosis and cytokinesis. Mitotic arrest or a cytokinesis defect can be introduced by depleting genes involved in mitotic progression or cytokinesis and cause visual changes that can be measured using markers for mitotic chromosomes (red) or total DNA (blue)

a broad spectrum of biological processes such as sister chromatid segregation, cytokinesis or the mitotic exit [18-20]. Such genetic interaction analyses have successfully been applied to further characterize hits from single gene screens $[19,21]$. Recently, genetic interaction analyses approach in yeast have increased the throughput to the genome-scale [22] and reported a close-to-complete coverage of all gene pairs by measuring $\sim 23 \times 10^{6}$ combinatorial knockouts [23]. To score genetic interactions in a metazoan model system, we developed an approach that uses systematic combinatorial RNAi in cultured Drosophila cells [24, 25].

Here, we discuss a recent study by our laboratory, which focuses on cell cycle-relevant phenotypic features in Drosophila cells and uses genetic interaction mapping to visualize functional networks underlying mitotic progression and cytokinesis [26]. This study characterized novel modulators by genome-wide high-content imaging RNAi screening, and structured the resulting 'hit' list using mitotic index- and nuclear area-focused genetic interaction analysis.

\section{Discussion}

\section{Distinct phenotypic features guide the detection of specific genetic interactions}

Cultured Drosophila cells have been used for genomescale loss-of function screens [9, 27-32] many of which investigating regulators of the cell cycle [9, 30-32]. Billmann and colleagues have screened the genome for cell cycle regulators by acquiring multiple phenotypic features through high-content imaging [26]. The comparison between cell count as a surrogate for fitness with the mitotic index of the population or the average nuclear area showed that the latter two features identified additional hits [26]. We selected roughly 300 genes affecting the mitotic index and nuclear size. Using genetic interaction analysis, networks were generated connecting genes that showed epistatic similarity when the mitotic index or nuclear area was measured. Often, genetic interactions were detected in the mitotic index but not when considering cell fitness only, thus providing additional, processspecific information for network generation (Fig. 2). Here, we discuss two analysis strategies that exploit this observation. Non-redundant multi-feature epistatic information enables inferring temporal functional relations between genes, while feature-specific epistatic profiles can reconstruct process-specific functional networks (Fig. 2).

\section{Multi-phenotype interactions can reconstruct directed hierarchies}

Recent work from our laboratory has identified epistatic relationships between genes building networks reflecting temporal order of gene function in processes such as the mitotic cell cycle [33]. Epistatic relationships were reconstructed by directed genetic interactions, indicating whether one gene repressed or amplified another genes effect (Fig. 3). This direction was inferred by comparing multi-feature phenotypic profiles of two genetically interacting genes with their combinatorial knockdown profile [33]. Phenotypic features were taken from cells stained

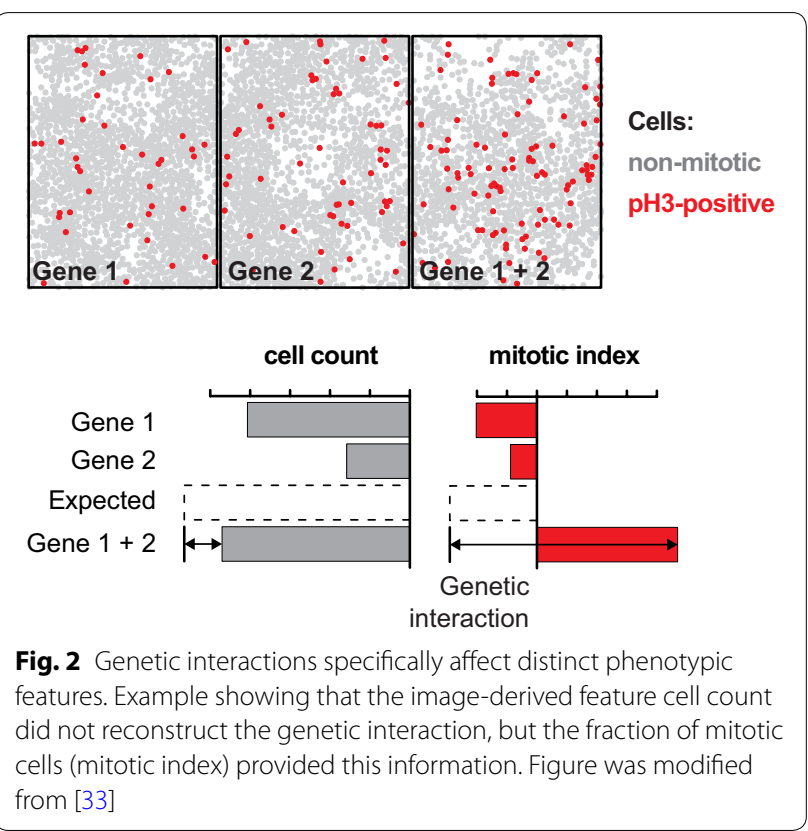




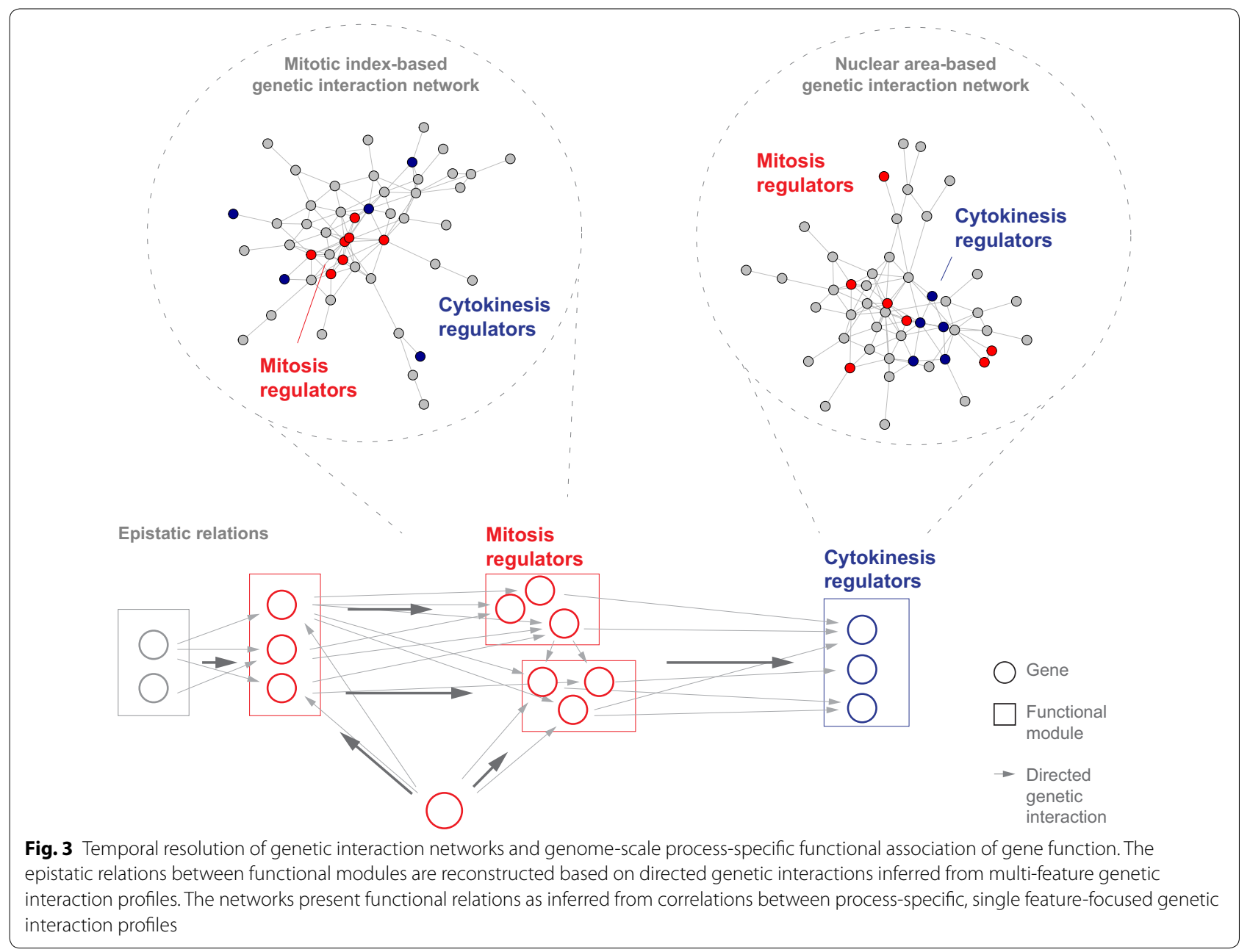

for their DNA content, the cytoskeletal component betatubulin as well as for the presence of $\mathrm{pH} 3$, and described population features as well as the shape and texture of cells [33].

For instance, this reconstructed an epistasis network between the components of functional modules of the mitotic cell cycle comprising structural modules such as the $\gamma$-tubulin ring complex, Condensin or Cohesin, regulatory modules such as the anaphase-promoting complex/cyclosome $(\mathrm{APC} / \mathrm{C})$ or the spindle assembly checkpoint (SAC), motor proteins (Dynein, Dynactin) and regulatory genes such as polo (Drosophila PLK1) [33]. This approach demonstrated that multi-parametric genetic interaction-based networks associate gene function and, in addition, provide epistatic relationships, thereby systematically visualizing functional relations between genes. Finally, features derived from the mitosis marker $\mathrm{pH} 3$ were highly informative for functional modules regulating mitosis, suggesting that feature-specific genetic interaction networks provide a snapshot of functional relations in the specific biological process.

\section{Phenotype-specific genetic interactions visualize process-specific networks}

To build functional networks of distinct cell cycle phases by genetic interaction analysis, we used high-content imaging of cell cycle features and a two-step screening approach: first, we screened a genome-wide RNAi library [34], measured genetic interaction profiles of selected genes and used this data to infer functional similarity [26] (Fig. 4). The genome-wide screen followed the rational that genes with a more pronounced depletion phenotype tend to genetically interact with a large fraction of genes $[33,35]$. The authors added genes with moderate phenotypic strength. To generate feature-specific genetic interaction networks, they used the mitotic index, the fraction of pH3-positive cells in the cell population, which serves as a proxy for mitotic progression. Mitotic index-based genetic interactions reconstructed regulatory modules of mitosis, but failed to group known components required for cytokinesis [26]. Clustering the latter components required the phenotypic feature nuclear size. This feature captures large, multi-nucleated cells, which arise due to 


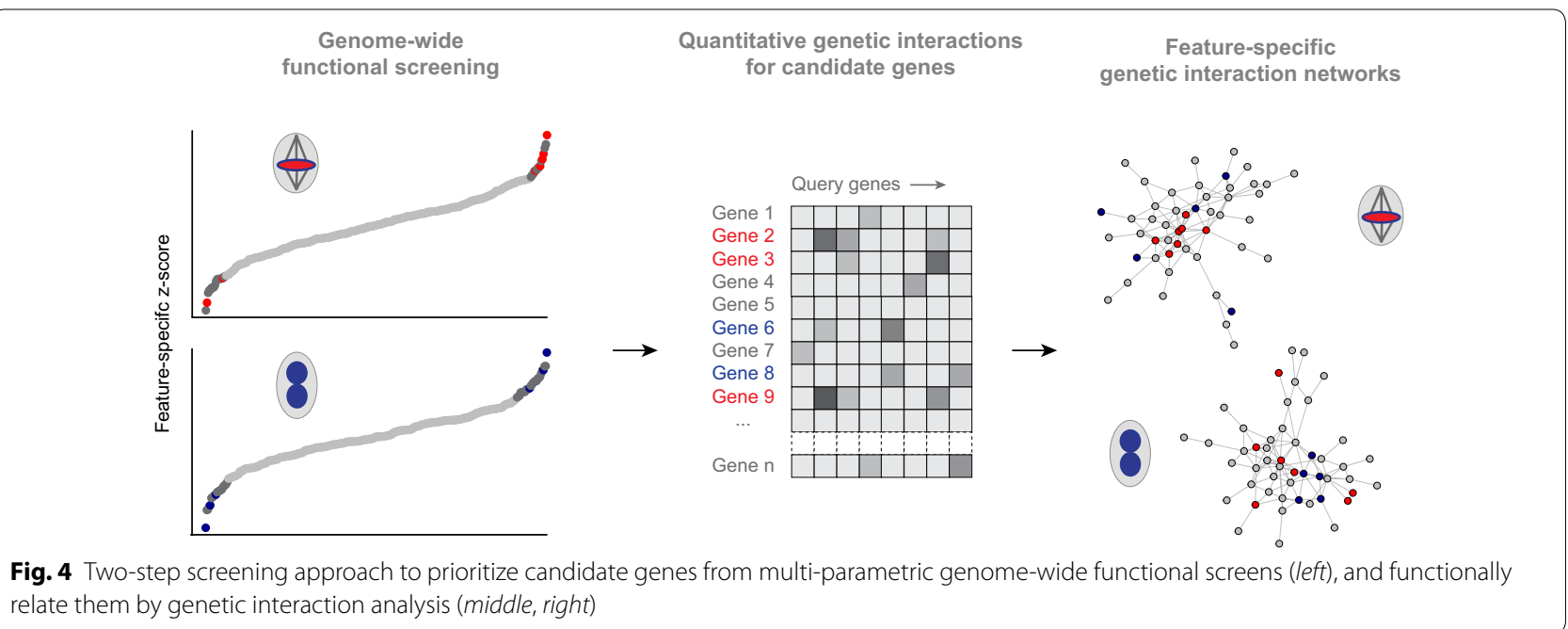

endoreduplication after failed cytokinesis [36]. Together, this approach generated genome-scale functional networks for specific cell cycle phases.

Those networks functionally assigned many potentially novel cell cycle regulators, which had often been described in processes not directly connected to cell cycle regulation. For those genes, their phenotypic strength alone insufficiently guided hypothesis generation. The second-line genetic interaction mapping approach deprioritized many of those hits, while suggesting hypothesis for others such as Golgi-resident components during mitotic progression [26].

\section{Conclusions}

Multi-feature imaging enables the visualization of epistatic relationships between genes by considering genetic interactions along the vector of phenotypic features such as cell count, mitotic index and nuclear area [5,33]. Moreover, genetic interactions affecting one processspecific feature capture a network of functional relations, zooming into a step of the causal chain in biological processes (Fig. 3).

Methodological rapid advances in CRISPR-based screens in mammalian systems and small molecule screens will require robust experimental and computational strategies to guide testable hypothesis. For example, a recent study in yeast generated various distinct phenotypic reporters by endogenously tagging various proteins with a GFP. The authors subsequently applied deep learning algorithms to the images to define cellular compartments and assess the response to genetic perturbations at multiple phenotypic levels [37]. Recently, a method integrated this high-content approach with a technique for systematic genetic interaction analysis in yeast [38], which will enable building networks illustrating functional relations in various biological processes.
Recent studies have also shown how to use imagebased screening for cellular phenotypes after treatment with small molecules in different genetic backgrounds to functionally group $\sim 1300$ pharmacologically active compounds [39]. Two-step screening approaches would allow to extent the number of screened small molecules by several orders of magnitude, while sensitively mapping the mode of action for pre-selected compounds.

Mapping gene function using genetic interactions has also been performed in mammalian cells [40-42]. Due to the larger genome size, two-step genetic interaction screening approaches provide an attractive strategy. While combinational RNAi face several challenges [43], more recent gene editing CRISPR/Cas9-based technologies enabled efficient and reliable gene perturbation across human cells [44]. In combination with a scRNAseq phenotypic readout, pooled CRISPR screens can be exploited to build process-focused genetic interaction networks in higher organisms [45]. Eventually, multi-step combinatorial gene depletion approaches will help building a systems view of biological processes such as the cell cycle [46] across genetic model systems.

\section{Abbreviations \\ RNAi: RNA interference; pH3: phosphorylated histone H3; APC/C: anaphase- promoting complex/cyclosome; SAC: spindle assembly checkpoint; GFP. green fluorescent protein; CRISPR: clustered regularly interspaced short palindromic repeats; scRNA-seq: single cell RNA sequencing.}

\section{Authors' contributions}

$\mathrm{MBi}$ and $\mathrm{MB}$ wrote the manuscript. Both authors have read and approved the final manuscript.

\footnotetext{
Author details

${ }^{1}$ German Cancer Research Center (DKFZ), Division Signaling and Functional Genomics and Heidelberg University, Department of Cell and Molecular Biology, Faculty of Medicine Mannheim, Im Neuenheimer Feld 580, 69120 Heidelberg, Germany. ${ }^{2}$ German Cancer Consortium (DKTK), 69120 Heidelberg, Germany. ${ }^{3}$ Present Address: Department of Computer Science and Engineering,
} 
University of Minnesota-Twin Cities, 200 Union St SE, Minneapolis, MN 55455, USA.

\section{Acknowledgements}

We would like to thank Thomas Horn and Bernd Fischer for critical discussions and comments on the manuscript.

\section{Competing interests}

The authors declare that they have no competing interests.

\section{Funding}

Work in the laboratory of M.B. is supported in part by an ERC Advanced Grant of the European Commission.

Received: 19 September 2016 Accepted: 26 December 2016

Published online: 06 January 2017

\section{References}

1. Nurse P. Genetic control of cell size at cell division in yeast. Nature. 1975:256:547-51.

2. Evans T, Rosenthal ET, Youngblom J, Distel D, Hunt T. Cyclin: a protein specified by maternal mRNA in sea urchin eggs that is destroyed at each cleavage division. Cell. 1983;33:389-96.

3. Gerlich D, Beaudouin J, Kalbfuss B, Daigle N, Eils R, Ellenberg J. Global chromosome positions are transmitted through mitosis in mammalian cells. Cell. 2003;112:751-64.

4. Gerlich D, Ellenberg J. Dynamics of chromosome positioning during the cell cycle. Curr Opin Cell Biol. 2003;15:664-71.

5. Boutros M, Heigwer F, Laufer C. Microscopy-Based High-Content Screening. Cell. 2015;163:1314-25.

6. Wei Y, Yu L, Bowen J, Gorovsky MA, Allis CD. Phosphorylation of histone H3 is required for proper chromosome condensation and segregation. Cell. 1999;97:99-109.

7. Prigent C, Dimitrov S. Phosphorylation of serine 10 in histone $\mathrm{H3}$, what for? J Cell Sci. 2003;116:3677-85.

8. Navarro FJ, Nurse P. A systematic screen reveals new elements acting at the G2/M cell cycle control. Genome Biol. 2012;13:R36.

9. Björklund M, Taipale M, Varjosalo M, Saharinen J, Lahdenperä J, Taipale J. Identification of pathways regulating cell size and cell-cycle progression by RNAi. Nature. 2006;439:1009-13.

10. Kittler R, Putz G, Pelletier L, Poser I, Heninger A-K, Drechsel D, et al. An endoribonuclease-prepared siRNA screen in human cells identifies genes essential for cell division. Nature. 2004;432:1036-40

11. Bakal C, Linding R, Llense F, Heffern E, Martin-Blanco E, Pawson T, et al. Phosphorylation networks regulating JNK activity in diverse genetic backgrounds. Science. 2008;322:453-6.

12. Fuchs F, Pau G, Kranz D, Sklyar O, Budjan C, Steinbrink S, et al. Clustering phenotype populations by genome-wide RNAi and multiparametric imaging. Mol Syst Biol. 2010;6:370.

13. Neumann B, Held M, Liebel U, Erfle H, Rogers P, Pepperkok R, et al. Highthroughput RNAi screening by time-lapse imaging of live human cells. Nat Methods. 2006;3:385-90.

14. Tong AH, Evangelista $M$, Parsons $A B, X u H$, Bader GD, Pagé N, et al. Systematic genetic analysis with ordered arrays of yeast deletion mutants. Science. 2001;294:2364-8.

15. Hartman JL, IV JLH, Garvik B, Hartwell L. Principles for the buffering of genetic variation. Science. 2001;291:1001-4.

16. Tong AHY, Lesage $G$, Bader GD, Ding H, Xu H, Xin X, et al. Global mapping of the yeast genetic interaction network. Science. 2004:303:808-13.

17. Collins SR, Miller KM, Maas NL, Roguev A, Fillingham J, Chu CS, et al. Functional dissection of protein complexes involved in yeast chromosome biology using a genetic interaction map. Nature. 2007;446:806-10.

18. Mayer ML, Pot I, Chang M, Xu H, Aneliunas V, KwokT, et al. Identification of protein complexes required for efficient sister chromatid cohesion. Mol Biol Cell. 2004;15:1736-45.

19. Huang D, Moffat J, Andrews B. Dissection of a complex phenotype by functional genomics reveals roles for the yeast cyclin-dependent protein kinase Pho85 in stress adaptation and cell integrity. Mol Cell Biol. 2002;22:5076-88

20. Nelson B, Kurischko C, Horecka J, Mody M, Nair P, Pratt L, et al. RAM: A conserved signaling network that regulates Ace $2 p$ transcriptional activity and polarized morphogenesis. Mol Biol Cell. 2003;14:3782-803.

21. Jorgensen P, Nishikawa JL, Breitkreutz B-J, Tyers M. Systematic identification of pathways that couple cell growth and division in yeast. Science. 2002;297:395-400.

22. Baryshnikova A, Costanzo M, Kim Y, Ding H, Koh J, Toufighi K, et al. Quantitative analysis of fitness and genetic interactions in yeast on a genome scale. Nat Methods. 2010;7:1017-24.

23. Costanzo M, VanderSluis B, Koch EN, Baryshnikova A, Pons C, Tan G, et al. A global genetic interaction network maps a wiring diagram of cellular function. Science. 2016;353.

24. Axelsson E, Sandmann T, Horn T, Boutros M, Huber W, Fischer B. Extracting quantitative genetic interaction phenotypes from matrix combinatorial RNAi. BMC Bioinform. 2011;12:342.

25. Horn T, Sandmann T, Fischer B, Axelsson E, Huber W, Boutros M. Mapping of signaling networks through synthetic genetic interaction analysis by RNAi. Nat Methods. 2011:8:341-6.

26. Billmann M, Horn T, Fischer B, Sandmann T, Huber W, Boutros M. A genetic interaction map of cell cycle regulators. Mol Biol Cell. 2016; 27(8):1397-407.

27. Müller P, Kuttenkeuler D, Gesellchen V, Zeidler MP, Boutros M. Identification of JAK/STAT signalling components by genome-wide RNA interference. Nature. 2005:436:871-5.

28. Boutros M, Kiger AA, Armknecht S, Kerr K, Hild M, Koch B, et al. Genomewide RNAi analysis of growth and viability in Drosophila cells. Science 2004:303:832-5.

29. Gesellchen V, Kuttenkeuler D, Steckel M, Pelte N, Boutros M. An RNA interference screen identifies inhibitor of apoptosis protein 2 as a regulator of innate immune signalling in Drosophila. EMBO Rep. 2005;6:979-84.

30. Kondo S, Perrimon N. A genome-wide RNAi screen identifies core components of the $\mathrm{G}_{2}-\mathrm{M}$ DNA damage checkpoint. Sci Signal. 2011;4:rs1.

31. Goshima G, Wollman R, Goodwin SS, Zhang N, Jonathan M, Vale RD, et al. Genes required for mitotic spindle assembly in Drosophila S2 cells. Science. 2007:316:417-21.

32. Bettencourt-Dias M, Giet R, Sinka R, Mazumdar A, Lock WG, Balloux F, et al Genome-wide survey of protein kinases required for cell cycle progression. Nature. 2004;432:980-7.

33. Fischer B, Sandmann T, Horn T, Billmann M, Chaudhary V, Huber W, et al. A map of directional genetic interactions in a metazoan cell. Elife. 2015;4:e05464

34. Horn T, Sandmann T, Boutros M. Design and evaluation of genome-wide libraries for RNA interference screens. Genome Biol. 2010;11:R61.

35. Costanzo M, Baryshnikova A, Bellay J, Kim Y, Spear ED, Sevier CS, et al. The genetic landscape of a cell. Science. 2010;327:425-31.

36. Zielke N, Querings S, Rottig C, Lehner C, Sprenger F. The anaphase-promoting complex/cyclosome (APC/C) is required for rereplication control in endoreplication cycles. Genes Dev. 2008:22:1690-703.

37. Chong YT, Koh JLY, Friesen H, Duffy K, Cox MJ, Moses A, et al. Yeast Proteome Dynamics from single cell imaging and automated analysis. Cell. 2015;161:1413-24.

38. Styles EB, Founk KJ, Zamparo LA, Sing TL, Altintas D, Ribeyre C, et al. Exploring quantitative yeast phenomics with single-cell analysis of DNA damage foci. Cell Syst. 2016:3:264-277.e10.

39. Breinig M, Klein FA, Huber W, Boutros M. A chemical-genetic interaction map of small molecules using high-throughput imaging in cancer cells. Mol Syst Biol. 2015;11:846

40. Laufer C, Fischer B, Billmann M, Huber W, Boutros M. Mapping genetic interactions in human cancer cells with RNAi and multiparametric phenotyping. Nat Methods. 2013;10:427-31

41. Roguev A, Talbot D, Negri GL, Shales M, Cagney G, Bandyopadhyay S, et al. Quantitative genetic-interaction mapping in mammalian cells. Nat Methods. 2013:10:432-7.

42. Vizeacoumar FJ, Arnold R, Vizeacoumar FS, Chandrashekhar M, Buzina A, Young JTF, et al. A negative genetic interaction map in isogenic cancer cell lines reveals cancer cell vulnerabilities. Mol Syst Biol. 2013;9:696 
43. Hart T, Moffat J. Scaling up the systematic hunt for mammalian genetic interactions. Nat Methods. 2013;10:397-9.

44. Hart T, Chandrashekhar M, Aregger M, Steinhart Z, Brown KR, MacLeod G, et al. High-resolution CRISPR screens reveal fitness genes and genotypespecific cancer liabilities. Cell. 2015;163:1515-26.
45. Dixit A, Parnas O, Li B, Chen J, Fulco CP, Jerby-Arnon L, et al. Perturb-seq dissecting molecular circuits with scalable single-cell RNA profiling of pooled genetic screens. Cell. 2016;167:1853-1866.e17.

46. Nurse P, Hayles J. The cell in an era of systems biology. Cell. 2011;144:850-4.

\section{Submit your next manuscript to BioMed Central} and we will help you at every step:

- We accept pre-submission inquiries

- Our selector tool helps you to find the most relevant journal

- We provide round the clock customer support

- Convenient online submission

- Thorough peer review

- Inclusion in PubMed and all major indexing services

- Maximum visibility for your research

Submit your manuscript at

www.biomedcentral com/submit 